\title{
Modelos experimentales para la investigación y el entrenamiento en trasplante renal
}

\author{
Sáenz Medina J, Asuero de Lis MS**, Correa Gorospe C***, Cuevas B***, Gómez Dos Santos V, \\ Linares Quevedo AI, Páez Borda A, Castillón Vela I, Marcén Letosa $\mathrm{R}^{* * * *}$, Pascual Santos J****, \\ Burgos Revilla FJ*. \\ Servicio de Urología. Hospital de Fuenlabrada. Madrid. *Departamento de Cirugía. Universidad de Alcalá. \\ Servicio de Urología. Hospital Ramón y Cajal. Madrid. **Servicio de Anestesiología y Reanimación. \\ Hospital Ramón y Cajal. Madrid. *** Unidad de investigación. Hospital Ramón y Cajal. Madrid. \\ ****Servicio de Nefrología. Hospital Ramón y Cajal. Madrid.
}

Actas Urol Esp. 2008;32(1):83-90

\section{RESUMEN}

MODELOS EXPERIMENTALES PARA LA INVESTIGACIÓN Y EL ENTRENAMIENTO EN TRASPLANTE RENAL

Introducción y objetivos: Se presenta una revisión sobre las diferentes características y el uso de los distintos modelos experimentales utilizados para el trasplante renal (TR). Esta revisión incluye las cualidades, así como sus semejanzas a los humanos, de las especies más frecuentemente utilizadas.

Se revisan las aportaciones de los diferentes modelos al entrenamiento de las diferentes técnicas quirúrgicas como la laparoscopia o la microcirugía.

Se repasan sus contribuciones al estudio y la investigación en campos como los efectos hemodinámicos o inmunológicos del neumoperitoneo, las técnicas de donante a corazón parado o las diferentes formas de preservación de los injertos.

Por último, se realiza una revisión de los diferentes modelos utilizados para la investigación de los distintos protocolos de inmunosupresión así como el xenotrasplante.

Palabras clave: Modelos experimentales. Trasplante renal.

\section{ABSTRACT}

EXPERIMENTAL MODELS FOR RESEARCH AND TRAINING IN RENAL TRANSPLANT

Introduction and objetives: An update on aspects and use of different experimental models applied in kidney transplant research is presented. This paper includes qualities, as long as similarities between most frequently used animal models and human clinical standards. Contributions of those models based on microsurgical or laparoscopic techniques are revised. The physiological consequences (hemodynamic, immunologic) of surgical technique (laparoscopy), applied in experimental models as long as non-heart beating organ donor models and organ preservation methods are also reviewed. Finally, an update of those models applied in research in prothocols of either immunosupression or xenotransplant is done.

Keywords: Experimental models. Renal transplantation.

\section{RECUERDO HISTÓRICO}

Las primeras referencias a modelos experimentales en torno al trasplante datan de la antigüedad en Grecia y Egipto donde eran habituales las representaciones gráficas de minotauros, sirenas, demonios o dioses (Fig. 1). Más tarde, en la época cristiana se describieron otras, como el trasplante realizado por San Cosme y San Damián en el 280 (Fig. 2).

Dejando aparte estas curiosas referencias producto de la imaginación de nuestros ancestros, a principios del siglo XX, se empezaron a desarrollar numerosos modelos experimentales de trasplante, siendo el riñón el más utilizado. El 7 de 


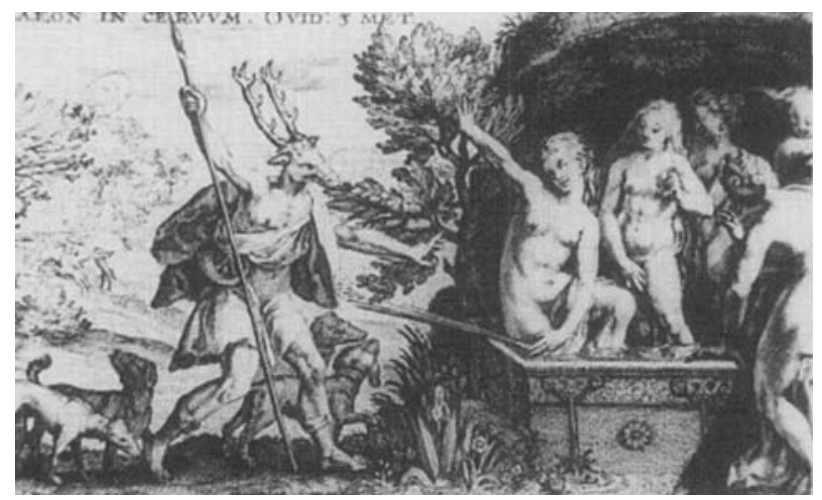

FIGURA 1. Grabado del siglo XVII. Biblioteque des arts decoratifs. París.

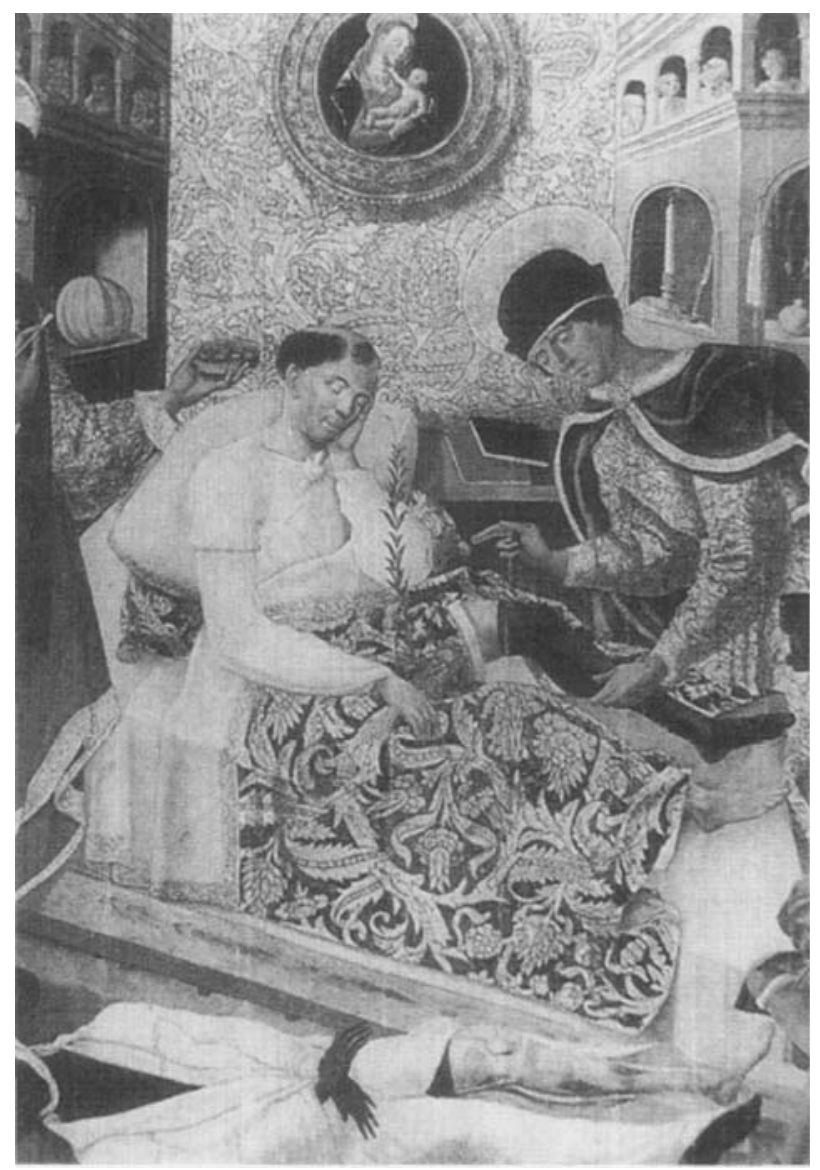

FIGURA 2. Fra Angelico: San Cosme y San Damián transplantando una pierna de cadáver en un sacristán.

Marzo de 1902, Ullmann ${ }^{1}$ (Fig. 3) realizó el primer trasplante de riñón en modelo experimental, realizando un alotrasplante de un perro al cuello de otro, anastomosando la arteria renal a la carótida y la vena renal a la yugular. El uréter lo abocó a la piel, y según refirió la audiencia de la época se produjo orina intraoperatoria, funcionando el injerto hasta 5 dias después del implante. Posteriormente intentó un xenotrasplante de cerdo en el codo de una mujer urémica, no consiguiendo terminar la cirugía por problemas técnicos.

Entre los trabajos más importantes, relativos al trasplante de órganos en modelo experimental, destacan los desarrollados por Alexis Carrell ${ }^{2}$, sobre anastomosis vasculares. Estos todavía están vigentes hoy en día y fueron merecedores de premio Nobel en 1904. Años más tarde, junto con el Dr. Charles Guthrie, Carrell realizó numerosas series de autotrasplante y de alotrasplante en perro $^{3}$.

Hacia finales del siglo XIX diferentes investigadores identificaron las bases de los grupos sanguíneos, la presencia de anticuerpos en suero (Elrich), la existencia del sistema del complemento (Bordet) y la presencia de células fagocíticas (Metchnikoff). En los años 40, Sir Peter Medawar, considerado padre de la inmunología del trasplante, estudió los factores de tolerancia en el receptor en un modelo murino de trasplante de piel, por lo que consiguió el premio Nobel en 1960.

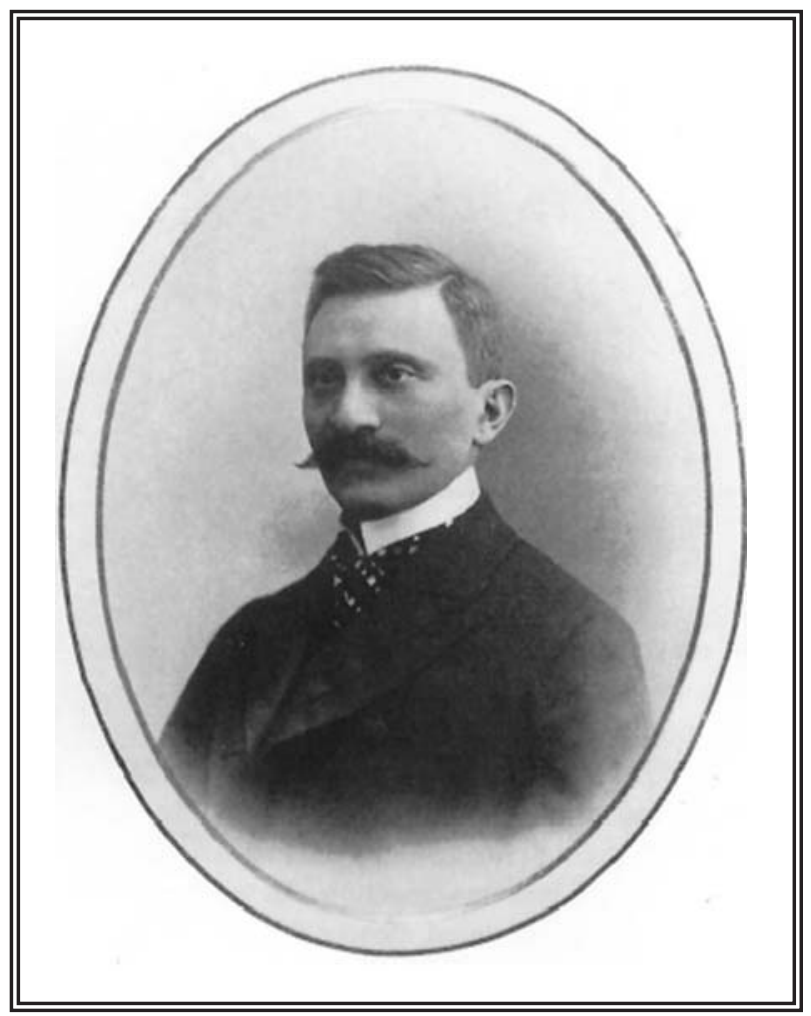

FIGURA 3. Emerich (Imre) Ullmann 1861-1937 (Universidad de Viena, Instituto de Historia de la Medicina). 
Posteriormente se demostró la utilidad de las radiaciones para provocar una inmunosupresión. Merril et al. en 1958, demostraron la capacidad de la irradiación linfática para prevenir el rechazo del trasplante renal (TR) en ratones y posteriormente en perros ${ }^{4}$.

A partir de entonces los modelos experimentales han contribuido al estudio de las sucesivas pautas de inmunosupresión así como al estudio y la investigación de las diferentes técnicas quirúrgicas, la preservación de los injertos o el xenotrasplante.

\section{CARACTERÍSTICAS DE LAS DIFERENTES ESPECIES ANIMALES Modelo porcino}

El cerdo utilizado como animal de experimentación en el campo del TR es ideal por su anatomía y fisiología tan similar al humano. Es un animal domesticable, de crecimiento rápido y reproducción numerosa. Sin embargo, hay que tener en cuenta que es de naturaleza muy lábil y sensible a las situaciones de estrés, por lo que su manejo debe ser cuidadoso para evitar la muerte del animal durante su preparación para la intervención.

El modelo experimental porcino simula fielmente el aparato cardiovascular humano tanto desde el punto de vista fisiológico como anatómico, dado que es similar en tamaño, distribución de la anatomía coronaria, y valores de presión arterial e índice cardíaco $^{5}$. Sin embargo, su mayor desarrollo del aparato digestivo, provoca diferencias respecto al humano en los estudios hemodinámicos realizados en el contexto la nefrectomía laparoscópica ${ }^{6}$.

En cuanto al riñón presenta menos cantidad de tejido adiposo renal que en el humano, perdiendo el efecto amortiguador de la fascia de Gerotta, que en nuestra especie, está descrito en estudios sobre flujo sanguíneo renal y laparoscopia $^{6}$. En cuanto a la configuración vascular renal Pereira-Sampaio et al. ${ }^{7}$ demuestran muchas similitudes entre ambas especies, postulándolo como el mejor modelo animal para cirugía renal.

\section{Modelo murino}

Los modelos murinos tienen la ventaja de ser animales de bajo costo, fácil cuidado y reproduc- ción; presentan un tiempo generacional muy corto, son muy prolíficos y se adaptan fácilmente a la vida en los bioterios, lo que permite controlar las variables ambientales en las experimentaciones.

Comparte con el hombre el privilegio de ser las especies de mamífero mejor estudiadas desde el punto de vista genético. Existe una cantidad enorme de líneas genéticamente definidas, además de miles de mutaciones. Es el único animal que posee sistemas eficientes de cultivo de células embrionarias pluripotenciales para generar quimeras, lo que permite la realización de mutaciones.

El primer TR en ratas está documentado en 1961 por Daniller et al. ${ }^{8}$ en 1961, con anastomosis termino-terminal arterial y venosa. En 1994 López Neblina et al. ${ }^{9}$ publicaron la realización de anastomosis vasculares para $\mathrm{TR}$ en ratas en menos de 6 minutos.

En cuanto a su aparato urinario, se ha demostrado la viabilidad del modelo murino para el estudio de la insuficiencia renal crónica, asî como el rechazo agudo y crónico ${ }^{10}$, ya que los hallazgos fisiopatológicos evidenciados se superponen con bastante fidelidad a los conocidos en humanos. Rollhaeuser et al. ${ }^{11}$ demostraron la similitud entre las anatomías vasculares renales murinas y humanas.

\section{Otros modelos (canino y primate no humano)}

E1 uso de modelos primates no humanos o caninos representa menos del $1 \%$ de la investigación en animales, si bien se han desarrollado modelos experimentales de ambas especies de TR.

Los modelos en primates ofrecen la ventaja de reproducir más fielmente el drenaje de la orina, que en los modelos cuadrúpedos. Se han realizado estudios ${ }^{12}$ de la anatomía vascular de algunas especies como el macaca fascicularis o el macaca mulata en los que se demuestra bastante similitud con el humano, aunque se evidencian algunas diferencias como la ausencia, en estas especies, de la linea de Brödel. Por el contrario, este modelo presenta la desventaja de ser caro y de dificil manejo y mantenimiento.

Los modelos caninos fueron muy usados en los inicios de la investigación experimental dada 
la fácil accesibilidad de los mismos, actualmente se utilizan menos por su mayor coste. Presentan la ventaja de disfrutar de mayor longevidad que las especies de granja y de ser una especie muy cercana al hombre. La raza más utilizada es el "beagle", debido a ser un perro de un tamaño intermedio, con carácter dulce, limpio, cuya crianza casi nunca presenta grandes dificultades.

\section{APORTACIONES AL ENTRENAMIENTO DE DIFERENTES TÉCNICAS QUIRÚRGICAS}

La utilización de modelos experimentales para el entrenamiento quirúrgico ha sido indispensable en el desarrollo del TR. El avance de la laparoscopia o de la microcirugia han sido sus aplicaciones fundamentales, sin embargo no se debe olvidar otras aplicaciones como el desarrollo de las anastomosis uretrovesicales o el ensayo de sustancias como los pegamentos biológicos.

De cualquier forma, las técnicas quirúrgicas urológicas en los animales de experimentación deben acogerse a las normas internacionales y los acuerdos del Consejo de Europa sobre protección de los animales empleados en experimentación animal y otros fines científicos, así como la reciente legislación nacional vigente (Directiva 86/609/CEE y R.D. 1201/2005).

\section{Laparoscopia}

La especie animal que con mayor frecuencia se utiliza como modelo experimental en laparoscopia urológica es la especie porcina, debido esencialmente a las semejanzas anatómicas del aparato urinario con el ser humano ${ }^{7}$ (similar tamaño del riñón, riñón multipapilar, uréteres de similar longitud).

En junio del año 90 Clayman et al. ${ }^{13}$ realizaron la primera nefrectomía vía laparoscópica en un modelo porcino por vía transperitoneal y, en diciembre del mismo año ${ }^{14}$, en humanos, mediante retroperitoneoscopia. En 1994 Gill et al. ${ }^{15}$ demostraron la viabilidad de la nefrectomía laparoscópica del donante en un modelo porcino. Como primer paso, se realizó primero una nefrectomía laparoscópica derecha en los 15 cerdos del estudio. Tres semanas después, una nefrectomía laparoscópica izquierda y autotrasplante convencional en 10 cerdos (grupo estudio); en el grupo control $(n=5)$ nefrectomía abierta y autotrasplante. Los niveles de creatinina a los siete y treinta días postoperatorios y el estudio anatomopatológico del injerto no demostraron diferencias significativas entre los grupos. Tampoco se evidenciaron diferencias en la tasa de complicaciones quirúrgicas entre ambos grupos.

En nuestro país G. Valdivia, uno de los pioneros de la laparoscopia en España desarrolló modelos experimentales para el aprendizaje de numerosas técnicas laparoscópicas ${ }^{16,17}$. Otros autores como Usón et al. apoyan el uso de esta especie como segundo escalón del modelo de enseñanza piramidal de aprendizaje laparoscópico ${ }^{18}$.

\section{Técnicas microquirúrgicas}

El desarrollo de técnicas de microcirugia ha sido fundamental para el desarrollo de las suturas vasculares del TR.

Se ha demostrado ${ }^{19}$, que la reparación de los vasos de injerto en el caso de lesiones vasculares, múltiples ramas, estenosis o aneurismas de la arteria renal no influyen en el funcionamiento a medio y a largo plazo del mismo. La realización de las mismas requieren de una gran destreza microquirúrgica vascular adquirida en modelos experimentales. La anastomosis de la carótida o la femoral en la rata, así como el TR se han postulado como modelos muy adecuados al respecto ${ }^{20}$.

\section{APORTACIONES A LA INVESTIGACIÓN EXPERIMENTAL}

Las principales líneas de investigación asociadas al TR en modelo animal se detallan a continuación:

\section{Modificaciones hemodinámicas, respiratorias y de los flujos vasculares asociadas a la nefrectomia laparoscópica de donante vivo}

La hiperpresión producida por el neumoperitoneo durante la laparoscopia, sobre la cavidad abdominal, condiciona una cascada de cambios hemodinámicos y respiratorios que han sido ampliamente estudiados a través de los modelos experimentales, especialmente porcinos ${ }^{6,21,22}$.

Se ha demostrado que el neumoperitoneo, en humanos, provoca descensos en el gasto cardíaco, aumento de las resistencias vasculares sisté- 
micas y ocasionales aumentos de la tensión arte$\mathrm{rial}^{23,24}$. En el modelo porcino, la mayoría de los estudios realizados ${ }^{6,21,22}$ no reproducen los mismos resultados. Se produce un aumento del GC, así como un descenso de las RVS; esto es probablemente debido a un mayor desarrollo del aparato digestivo, así como una mayor capacidad del ventrículo izquierdo porcino para asumir los volúmenes procedentes de la aurícula izquierda. Esta mayor capacidad probablemente no se deba a un mayor desarrollo intrínseco del ventrículo sino a una menor repercusión de la hiperpresión abdominal sobre el tórax por la diferente configuración de la pared abdominal del cerdo ${ }^{6}$.

En cuanto al aparato respiratorio, el modelo porcino sí reproduce con fidelidad la cirugía laparoscópica en humanos ${ }^{6}$. El aumento de los niveles de $\mathrm{CO}_{2}$ producido por la hiperpresión abdominal y por la difusión del gas a través del peritoneo, deben ser controlados por el anestesista aumentando el volumen y la frecuencia respiratoria, para favorecer la eliminación del mismo ${ }^{25,26}$.

Los flujos vasculares también se ven afectados por el neumoperitoneo. Nuestro grupo ha demostrado un aumento del flujo carotideo producido por el neumoperitoneo, junto con un descenso del flujo hepático tanto a nivel de la arteria hepática, como a nivel portal ${ }^{6}$. En cuanto al flujo sanguíneo renal, si bien inicialmente se demostró un descenso asociado al neumoperitoneo ${ }^{27}$, posteriormente se evidenció la corrección del mismo mediante el uso de hidroxietilalmidón en la reposición hidroelectrolítica y la disminución de la presión intrabdominal utilizada ${ }^{6}$, tal y como previamente habian demostrado otros autores con modelos experimentales muy similares ${ }^{28,29}$.

\section{Marcadores de agresividad quirúrgica asociados al TR}

Una de las ventajas más importantes asociadas al abordaje laparoscópico de la nefrectomía de donante vivo es su menor agresividad, lo que implica una disminución del dolor postoperatorio, de las estancias hospitalarias, y de la incorporación a la vida activa.

Para valorar correctamente el grado de repercusión clínica que tiene la técnica laparoscópica sobre el paciente y cuantificar su agresividad, se han propuesto modelos porcinos en los que se cuantifican marcadores de estrés postquirúrgico como las citocinas o los reactantes de fase aguda. La medición de éstos ha demostrado que la cirugía laparoscópica provoca un menor estrés, proporcionando un claro beneficio para el paciente especialmente durante el postoperatorio inmediato ${ }^{30}$. El modelo porcino se ha validado para el estudio del comportamiento del sistema inmunitario bajo la influencia de la cirugía laparoscópica ${ }^{30,31}$.

\section{Modelos experimentales de donante a corazón parado}

La necesidad de conseguir más injertos renales, debido al incremento constante de los enfermos en diálisis, y a la ampliación de las indicaciones de TR, ha conducido al estudio de viabilidad de injertos renales de donantes a corazón parado, cuya diferencia más importante con los donantes clásicos es el aumento del tiempo de isquemia caliente ${ }^{32}$. Se ha estimado que el periodo de isquemia caliente máximo, para que un injerto, sea viable es de 30 minutos, si bien se han realizado técnicas, como los estudios del grupo del Hospital Clinic de Barcelona en los que parece alargarse este tiempo ${ }^{33}$.

Para estudiar este tipo de donantes, se han utilizado modelos animales tanto en ratón ${ }^{34}$ como en cerdo ${ }^{35}$, intentado reproducir el efecto de isquemia reperfusión producido. El periodo de isquemia caliente genera sustancias vasoactivas como el óxido nitroso y posteriormente durante el periodo de reperfusión se liberan radicales libres de oxígeno que aumentan las resistencias vasculares. Estos cambios pueden incidir sobre la función inmediata del injerto. El estudio de estos cambios, en modelos animales, permiten experimentar diferentes técnicas como las máquinas de perfusión de los injertos o las diferentes soluciones de preservación para tratar de minimizar estos efectos.

\section{Máquinas de perfusión y soluciones de preservación de injertos renales}

Ante la necesidad de mejorar la preservación de los órganos, se han desarrollado dos líneas de investigación en animales a propósito de este problema, por un lado se han experimentado diversas máquinas de perfusión, y por el otro múltiples soluciones de preservación. 
Desde hace más 30 años se inició la experimentación de máquinas de perfusión con animales, inicialmente perros ${ }^{36}$. Se comenzaron usando bombas de rodillos, inicialmente desarrolladas a partir de las máquinas de diálisis. Posteriormente se han ido elaborando otros sistemas como los de vacío asociados a sistemas informáticos ${ }^{37}$. Paralelamente se ha debatido extensamente el uso de máquinas pulsátiles o de flujo contínuo ${ }^{36}$ y se han desarrollado sistemas de predicción de función inmediata del injerto en función de las presiones halladas durante la perfusión así como de los metabolitos analizados en la solución perfundida ${ }^{38}$.

De la misma forma, se han experimentado diversas soluciones de preservación. En 1967 Belzer et al. ${ }^{39}$ publicaron modelos en perros en los que se perfundía plasma o sangre oxigenada. Posteriormente Collins et al. ${ }^{40}$ demostraron que la inmersión de los injertos en suero salino helado era capaz de mantenerlos viables durante 12 horas, y desarrollaron una solución similar al líquido intracelular, consiguiendo preservar los riñones en modelo canino durante 30 horas. Esta contenía, además del suero salino, procaína, heparina, glucosa y fenoxibenzamina ${ }^{41}$. Un año mas tarde se añadió a esta solución albúmina y se denominó la solución de Collins ${ }^{42}$.

A finales de la década de los 80 , Belzer et al. ${ }^{43}$ desarrollaron la solución de preservación de la universidad de Wisconsin también en modelos experimentales de perros, que introducía tres nuevas diferencias, la osmolaridad no se mantenía a base de glucosa metabólicamente activa sino a base de sustancias inertes como el lactobionato y la rafinosa, el uso del coloide hidroxietilalmidón y los captadores de radicales libres como el glutation y la adenosina.

Posteriormente, se han desarrollado otras soluciones como el HTK (Histidin-TryptophanKetoglutarat) ${ }^{44}$ o el celsior, en modelos porcinos, sin conseguir mejorar sustancialmente el winconsin, siendo éste último de referencia.

\section{Modelos experimentales e inmunosupresión en el TR}

Como previamente se ha expuesto, ya en los inicios del trasplante se descubrió que el uso de ciertos agentes como la radiación de los ganglios linfáticos mejoraba notablemente la supervivencia de los injertos ${ }^{4}$. Desde 1975 , se ha mejorado la supervivencia del injerto renal desde un 50 hasta un $90 \%$, con una supervivencia del riñón, al año del TR, en torno al 93\%.

Se han desarrollado diversos modelos experimentales sobre todo murinos, porcinos y en primates ${ }^{45}$ para evaluar la eficacia y los mecanismos de acción de las diferentas pautas inmunosupresoras, así como modelos para analizar los efectos secundarios de las distintas drogas.

La evaluación del mecanismo de acción de las drogas inmunosupresoras, se realiza a través de modelos de rechazo o nefropatía crónica, generalmente murinos con líneas genéticamente tratadas como las "Wistar furth" (RT1u) o las DA "RT1a". Estos modelos reproducen histológica y funcionalmente la nefropatía crónica del injerto una vez realizado el TR, posibilitando la evaluación del mecanismo de acción y de los efectos secundarios de los inmunosupresores ${ }^{10}$. Sin embargo, no existen referencias bibliográficas en relación a modelos experimentales en TR para analizar las complicaciones quirúrgicas, habiendo sido realizados la mayoría de estos estudios en el ámbito clínico.

\section{Xenotrasplante}

La primera cita con carácter "científico" documentada en el ámbito del xenotrasplante corresponde a la realización de diversas transfusiones de sangre de animal al hombre en 1628 por Colle de Belluno de Padua, en 1654 por Francesco Folli y en 1665 por Richard Lower de Londres ${ }^{46}$.

En 1906 se comunica el primer xenotrasplante "real" según los términos con el que lo conocemos hoy día. En ese año, Mathieu Jaboulay injerta el riñón de un cerdo a una mujer afectada por un síndrome nefrótico y el de una cabra en el codo de una mujer de 50 años. En ambos casos, los resultados fueron negativos debido a una hipercoagulación de la sangre, que en el fondo no era más que un rechazo hiperagudo ${ }^{46}$.

En 1996, White et al. ${ }^{47}$, publica los primeros resultados esperanzadores utilizando cerdos modificados genéticamente. La estrategia utilizada consistió en introducir un gen humano en el organismo de estos animales con el fin de frenar los mecanismos de respuesta natural que activan 
el rechazo hiperagudo. Se trasplantó con éxito el corazón de cerdos modificados genéticamente en primates y se comprobó como los órganos superaban el rechazo hiperagudo. El futuro inmediato del xenotrasplante pasa por profundizar en diferentes lineas de investigación.

En nuestro país González Martín et al. ${ }^{48}$ han realizado estudios de xenotrasplante renal de cerdo transgénico a babuino, consiguiendo superar el rechazo hiperagudo pero no así el rechazo vascular o humoral agudo.

Se apuntan como aspectos clave el desarrollo de nuevas pautas de inmunosupresión basadas o no en los regímenes actuales para la profilaxis del rechazo, y la aplicación de técnicas de ingeniería genética que permitan modificar la respuesta del órgano trasplantado.

\section{REFERENCIAS}

1. Ullmann E: Experimentelle Nierentransplantation. Wien Klin Wochenschr 1902; 11: 281.

2. Carrel A. La technique operatoire des anastomoses vasculaires et la transplantation des visceres. Lyon Med 1902; 98: 859.

3. Carrel A. Results of the transplantation of blood vessels. organs and limbs [reprint; originally published November 14, 1908]. JAMA. 1983;250(7):944-953.

4. Murray JE, Merrill JP, Dammin GJ, Dealy JB JR, Walter CW, Brooke MS, et al. Study of transplantation immunity after total body irradiation: Clinical and experimental investigation. Surgery. 1960;48:272-284.

5. Kaklamanos IG, Condos S, Merrell RC. Time-related changes in hemodynamic parameters and pressure-derived indices of left ventricular function in a porcine model of prolonged pneumoperitoneum. Surg Endosc. 2000;14(9): 834-838.

6. Sáenz J. Modificaciones hemodinámicas y marcadores de estrés postquirúrgico inducidos por el neumoperitoneo en la cirugía laparoscópica urológica. Modelo Experimental. Alcalá de Henares. 2006

7. Pereira-Sampaio MA, Favorito LA, Sampaio FJ. Pig kidney: Anatomical relationships between the intrarenal arteries and the kidney collecting system. Applied study for urological research and surgical training. J Urol. 2004;172(5 pt1):2077-2081.

8. D'Silva M, Gittes RF, Wolf P, Pirenne J, Munger K, Pascual $\mathrm{J}$, et al. Rat kidney transplantation update with special reference to vesical calculi. Microsurgery. 1990;11(2):169176 .

9. Lopez-Neblina F, Toledo-Pereyra LH, Suzuki S. Ultrarapid orthotopic technique for renal transplantation in the rat. Microsurgery. 1994;15(4):274-278.

10. Savikko J, von Willebrand E. Sirolimus attenuates chronic allograft nephropathy in an experimental rat kidney transplantation model. Transp Proc. 2006;38(8):2699-2700.

11. Rollhaeuser H, Kriz W, Heinke W. The vascular system of the rat kidney. Z Zellforsch Mikrosk Anat. 1964;64:381403.
12. Horacek MJ, Earle AM, Gilmore JP, et al. The renal vascular system of the monkey: a gross anatomical description. J Anat. 1987;153:123-137.

13. Clayman RV, Kavoussi LR, Long SR, et al. Laparoscopic nephrectomy. Initial report of pelvicoscopic organ ablation in the pig. J endourol. 1990;4:247.

14. Clayman RV, Kavoussi LR, Soper NJ, Dierks SM, Merety KS, Darcy MD, et al. Laparoscopic nephrectomy (letter). N Engl J Med. 1991;324(19):1370-1371.

15. Gill IS, Carbone JM, Clayman RV, Fadden PA, Stone MA, Lucas BA, et al. Laparoscopic live donor nephrectomy. J endourol. 1994;8(2):143-148.

16. Valdivia Uría JG, Viloria González A, Rodríguez Gómez J, Valle Gerhold J, Martínez Sañudo MJ, Whyte Orozco A, et al. Cistoprostatovesiculectomía y ureterosigmoidostomía laparoscópica. Modelo experimental. Actas Urol Esp. 1992; 16(7):592-598.

17. Valdivia Uría JG, Viloria González A, Rodríguez Gómez J, Martínez Sañudo MJ, Whyte Orozco A, Valle Gerhold J, et al. Nefrectomía laparoscópica. Modelo experimental. Actas Urol Esp. 1992;16(2):169-174.

18. Usón Gargallo J, Sánchez Margallo FM, Díaz-Güemes Martín-Portugués I, Loscertales Martín de Agar B, Soria Gálvez F, Pascual Sánchez-Gijón S. Modelos experimentales en la cirugía laparoscópica urológica. Actas Urol Esp. 2006;30(5):443-450.

19. Galmés I, Burgos FJ, Rodríguez-Luna JM, Fernández E, Puras E, Ortiz F, et al. "Ex situ" reconstructive surgery of the renal arteries. Actas Urol Esp. 1996;20(1):30-36.

20. Korber KE, Kraemer BA. Heterotopic renal transplantation in the rat: an advanced microsurgical training exercise. Microsurgery. 1988;9(4):286-291.

21. Ortega AE, Richman MF, Hernandez M, Peters JH, Anthone GJ, Azen S, et al. Inferior vena caval blood flow and cardiac hemodynamics during carbon dioxide pneumoperitoneum. Surg Endosc. 1996;10(9):920-924.

22. Bannenberg JJ, Rademaker BM, Froeling FM, Meijer DW. Hemodynamics during laparoscopic extra- and intraperitoneal insufflation An experimental study. Surg Endosc. 1997;11(9):911-914.

23. Chiu AW, Chang LS, Birkett DH, Babayan RK. The impact of pneumoperitoneum, pneumoretroperitoneum, and gasless laparoscopy on the systemic and renal hemodynamics. J Am Coll Surg. 1995;181(5):397-406.

24. Joris JL, Noirot DP, Legrand MJ, Jacquet NJ, Lamy ML. Hemodynamyc changes during laparoscopic cholecystectomy. Anest Analg. 1993;76(5):1067-1071.

25. Gutt CN, Oniu T, Mehrabi A, Schemmer P, Kashfi A, Kraus $\mathrm{T}$, et al. Circulatory and respiratory complications of carbon dioxide insufflation. Dig Surg. 2004;21(2):95105.

26. McDermott JP, Regan MC, Page R, Stokes MA, Barry K, Moriarty DC, et al. Cardiorespiratory effects of laparoscopy with and without gas insufflation. Arch Surg. 1995;130(9): 984-988.

27. Linares Quevedo AI. Estudio comparativo de la función del trasplante renal con donante vivo mediante nefrectomía convencional y laparoscópica. Influencia del síndrome de isquemia repercusión. Modelo experimental. Madrid: 2005.

28. London ET, Ho HS, Neuhaus AM, Wolfe BM, Rudich SM, Perez RV. Effect of intravascular volume expansion on renal function during prolonged $\mathrm{CO} 2$ pneumoperitoneum. Ann Surg. 2000;231(2):195-201. 
29. Junghans T, Böhm B, Gründel K, Schwenk W, Müller JM. Does pneumoperitoneum with different gases, body positions, and intraperitoneal pressures influence renal and hepatic blood flow?. Surgery. 1997;121(2):206-211.

30. Burgos FJ, Linares A, Pascual J, Marcen R, Villafruela J, Zamora J, et al: Modifications of renal blood flow and serum interleukin levels induced by laparoscopic and open living donor nephrectomies for kidney transplant: an experimental study in pigs. Transplant Proc. 2005;37(9): 36763678.

31. Hiki N, Shimizu N, Yamaguchi H, Imamura K, Kami K, Kubota $\mathrm{K}$, et al. Manipulation of the small intestine as a cause of the increased inflammatory response after open compared with laparoscopic surgery. Br J Surg. 2006;93 (2):195-204.

32. Sánchez-Fructuoso AI, Prats D, Torrente J, Pérez-Contín MJ, Fernández C, Alvarez J, et al. Renal Transplantation from Non-Heart Beating Donors: A Promising Alternative to Enlarge the Donor Pool. J Am Soc Nephrol. 2000;11(2): 350-358.

33. Mendes DR, Gibanel R, Capdevila S, Ruiz A, Alcoberro J, Calatrava P, et al. Strategies to Enhance Organ Viability in a Non-Heart-Beating Donor Extracorporeal Recirculation Transplant Model in Pigs. Transp Proc. 2003;35(2):845846.

34. Montón S, Herrera J, Ferrer JV, Guerrero D, Balén E, Lera JM. Experimental Model of Non-Heart-Beating Donors: Oxidative Stress Metabolism in Kidney After Cardiac Arrest (30 Minutes of Warm Ischemia) and Reimplantation 24 Hours Later. Transp Proc. 1999;31(6):2350-2351.

35. Alcoberro J, Alcaraz A, Alvarez-Vijande R, Calatrava P, Luque P, Rodriguez A, et al. Experimental Kidney Transplantation in Pigs From Non-Heart-Beating Donors: Evaluation of Renal Artery Flow. Transp Proc. 1999;31(6): 2348-2349.

36. Grundmann R, Pitschi H, Berr F, Pichlmaier H. Nonpulsatile vs. pulsatile canine kidney perfusion. Surgery. 1974;75(2):178-184.

37. Lledó García E, Hernández Fernández C, Llorente Abarca C, del Cañizo López JF. Efectos hidrodinámicos y bioquímicos de la perfusión hipotérmica de riñón aislado según el tipo de bomba utilizada. Actas Urol Esp. 2002;26 (3):182-189.
38. Baicu SC, Taylor MJ, Brockbank KG. The role of preservation solution on acid-base regulation during machine perfusion of kidneys. Clin Transplant. 2006;20(1):113-121.

39. Belzer FO, Ashby BS, Dunphy JE. 24-Hour and 72 Hour preservation of Canine Kidneys. Lancet. 1967;2(7515):536538.

40. Collins G, Shugarman MB, Terasaki PI, et al. Kidney Preservation for Tranplantation. Transplantation 1969;8: 821.

41. Collins GM, Bravo-Shugarman M, Terasaki PI. Kidney preservation for transportation. Initial perfusion and 30 hours' ice storage. Lancet. 1969;2(7632):1219-1222.

42. Terasaki PI: Transportation and storage. Transplant Newsletter 1970.

43. Ploeg RJ, Goossens D, McAnulty JF, Southard JH, Belzer FO. Successful 72-hour cold storage of dog kidneys with UW solution. Transplantation. 1988;46(2):191-196.

44. Manekeller S, Leuvenink H, Sitzia M, Minor T. Oxygenated machine perfusion preservation of predamaged kidneys with HTK and Belzer machine perfusion solution: an experimental study in pigs. Transp Proc. 2005;37(8):3274-3275.

45. Jonker M, Ringers J, Ossevoort MA, Slingerland W, van den Hout Y, Haanstra K, et al. Long-term kidney graft survival by delayed $\mathrm{T}$ cell ablative treatment in rhesus monkeys. Transplantation. 2002;73(6):874-880.

46. Toledo-Pereyra LH , Lopez-Neblina F. Xenotransplantation: a view to the past and an unrealized promise to the future. Exp Clin Transplant. 2003;1(1):1-7.

47. White DJ , Yannoutsos N. Production of pigs transgenic for human DAF to overcome complement-mediated hyperacute xenograft rejection in man. Res Immunol. 1996;147(2): 88-94.

48. González Martín M, García Buitrón J, Alonso Hernández A, Centeno Cortés A, López Peláez E, Vázquez Martul E, et al. Xenotrasplante renal cerdo hDAF- babuino. Experiencia y revisión. Actas Urol Esp. 2004;28(3):161-174.

Correspondencia autor: Dr. J. Sáenz Medina Servicio de Urología. Hospital de Fuenlabrada Camino del Molino, s/n - 28942 Fuenlabrada (Madrid). Tel.: 916006000

E-mail autor: javiersaenzmedina@yahoo.es Información artículo: Original 